\title{
Wavelength tuning in spherical liquid dye lasers by controlling the cavity $Q$ values
}

\author{
Hiroshi Taniguchi \\ Department of Electrical and Electronic Engineering, Faculty of Engineering, Iwate University, 4-3-5 Ueda, \\ Morioka 020, Japan \\ Humihiro Tomisawa \\ Department of Electrical Engineering, Faculty of Engineering, Iwate University, 4-3-5 Ueda, Morioka 020, \\ Japan
}

(Received 25 July 1994; accepted for publication 24 October 1994)

A method for wavelength tuning in spherical liquid dye lasers is reported, due to controlling cavity $Q$ values by injection of scattering particles. The cavity $Q$ value of the sphere dye lasers is controlled by varying the quantity of poly(methyl methacrylate) spheres in the spherical liquid laser solution. Results of the tuning are explained in terms of a general wavelength dependence determined by gain/loss characteristics in the microsphere lasers. (C) 1994 American Institute of Physics.

A number of recent papers have reported lasing from dye-doped micrometer-sized spheres; liquid droplets ${ }^{1-3}$ and solid spheres. ${ }^{4-6}$ Small spheres, such as dielectrics, act as high- $Q$ optical cavities; optical feedback is provided by light waves that totally internally reflect at the sphere-surrounding media interface and fold back on themselves. A variety of nonlinear effects such as stimulated Raman scattering (SRS) $)^{2,7-9}$ has also been observed when liquid micrometersized droplets are irradiated by intense laser beams. These lasing and SRS emissions occur at discrete wavelengths that correspond to morphology-dependent resonances (MDRs) [or whispering-gallery mode (WGM)] of the sphere. The spontaneous transition rates of fluorescent or Raman-active molecules are enhanced by MDRs of the spherical microcavity, which provides the optical feedback needed to support lasing and SRS within the spontaneous bandwidths.

Previous investigations of dye-doped solid microsphere lasers that are due to MDRs (or WGM) ${ }^{4-6}$ have demonstrated their usefulness for practical application; however, it has been experimentally suggested that a more efficient laser performance that is due to MDRs is more possible in liquid spheres than in solid spheres because of lower optical losses, such as adsorption losses of dye molecules onto the solid spheres, and because of the higher- $Q$ values of the much smoother liquid spheres. ${ }^{10,11}$ From the viewpoint that microdroplets may contain particulates, lasing behavior was reported recently in liquid droplets that contain a fluorescent dye solution and either fluorescent $\mathrm{sol}^{12}$ or undoped latex-sol ${ }^{13}$ particles; further, SRS behavior was also reported when latex sol was added to either pure or rhodamine 6Gdye-doped ethanol droplets. ${ }^{14}$ In spite of such a great number of investigations on nonlinear optical devices using spheres, to our knowledge, no techniques for wavelength tuning have been discussed to the present. Realization of tunable microsphere dye lasers with high- $Q$ values (i.e., with low thresholds) may promise developments of nonlinear optical microdevices.

In this letter we report the first experiments, to our knowledge, for wavelength tuning of droplet dye lasers. The experiments for the wavelength tuning are carried out with injecting scattering particles into the droplet dye laser. The lower cavity $Q$ value in the presence of scattering particles leads to lasing at shorter wavelengths, corresponding to the larger population inversion (gain) to reach the lasing threshold. The results of the wavelength tuning are explained, in principle, based on a cavity $Q$ value in terms of a general wavelength dependence of dye lasing determined by gain/ loss characteristics, in the droplet dye lasers.

The experimental arrangement, is similar to those described previously, ${ }^{10,11}$ however, the present experiment employs a frequency-doubled, $Q$-switched Nd:YAG laser (Nd:YAG) second-harmonic (SH); $532 \mathrm{~nm}$ with $\sim 3 \mathrm{~mJ}$ pulse energy and $\sim 10 \mathrm{~ns}$ pulse duration instead of a pulsed $\mathrm{UV} \mathrm{N}_{2}$ laser $(337.1 \mathrm{~nm})$. Green light from the Nd:YAG SH $(532 \mathrm{~nm})$ is focused onto dye-doped liquid spheres that scatter elastic $(532 \mathrm{~nm})$ and inelastic (redshifted dye lasing) light into an optical multichannel analyzer. The weakly focused pumpbeam size was large compared to the dye-doped sphere [rhodamine 6G (RH-6G) or rhodamine 101 (RH-101)]. Spectra of light emitted from the dye-doped sphere are collected at $90^{\circ}$ to the incident pump beam and are analyzed with an optical multichannel analyzer mounted on a microscope. Fine aerosol particles (2-3 $\mu \mathrm{m}$ diam) of ethanol-containing dye liquids are successively ejected from a modified atomizer and grown to proper-sized droplets (of the order of 200 $\mu \mathrm{m}$ in diameter) around a fine horizontally strung tungsten fiber ( $\sim-7 \mu \mathrm{m}$ diam). The dye-doped droplets are made from a glycerol-ethanol mixture with a composition ratio of $\sim 5: 1$ by volume. The 5:1 dilution is used to produce adequate viscosity because glycerol alone has a high viscosity and to produce droplet-size stability because ethanol alone has a higher evaporation rate.

An example of a microphotograph of an RH-6G- (the upper panel in Fig. 1) or RH-101-doped single droplet (the lower panel in Fig. 1) is shown in Fig. 1, in which with/ without cases of the seeding of artificial scattering spherical particles [poly(methyl methacrylate) (PMMA) with $\sim 50-\mu \mathrm{m}$ diam] into the droplet are included. Figure 1 comes from the same single droplet ( $\sim 10^{-3} \mathrm{M}$ concentration in both dyes) by the Nd:YAG SH pumping, without seeding, with seeding 

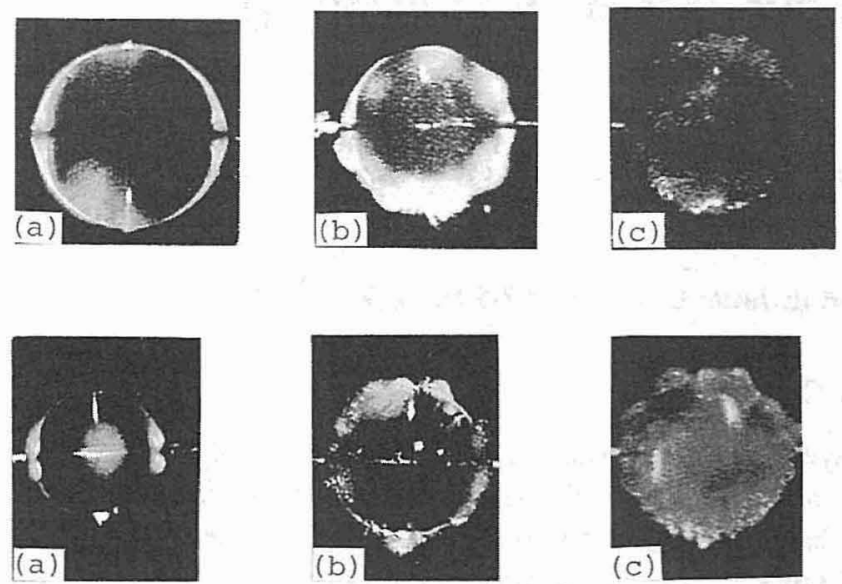

FIG. 1. Microphotograph of RH-6G- (upper) and RH-101- (lower) doped single droplets ( $\sim 10^{-3} \mathrm{M}$ concentration in both dyes) by Nd:YAG SH (532 $\mathrm{nm})$ pumping; (a) without PMMA seeding, (b) with PMMA seeding $(\sim 1 /$ 10-PMMA volume of the initial droplet volume), and (c) with more PMMA seeding ( $\sim 1 / 2$ volume). Orange (red), yellow (orange), and green are seen, in the RH-6G (RH-101) dye, in (a), (b) and (c), respectively.

( 1/10-PMMA volume of the initial droplet volume), and with more seeding ( $\sim 1 / 2$ volume), in Fig. 1(a) orange (red), Fig. 1(b) yellow (orange), and Fig. 1(c) green, in the RH-6G (RH-101) dye, respectively. The concentration of PMMA was estimated from estimating the total numbers of PMMA (with diameter of $\sim 50 \mu \mathrm{m}$ and specific gravity of 1.19), by measuring the total weights of PMMA.

In the spectral results exhibited in Figs. 2 and 3, the SH is the elastic spectrum of the pump Nd:YAG SH light (532 $\mathrm{nm})$ and RO is the oscillation spectra emitted from the RH-6G (Fig. 2) or RH-101 (Fig. 3) dye.

The single-shot RO spectral shift is shown in Fig. 2, of light emitted from the externally seeded droplets. The pumping intensities are held nearly constant with full highamplitude above the lasing threshold. Figure 2(a) is the lasing spectrum from the RH-6G-doped droplet $\left(\sim 10^{-3} \mathrm{M}\right.$ concentration, $d \approx 210 \mu \mathrm{m}$ diam) alone (i.e., without PMMA seeding), Figs. 2(b), 2(c), and 2(d) $(\sim 1 / 20,1 / 10$, and 1/5) correspond to the lasing spectrum with gradual increas-

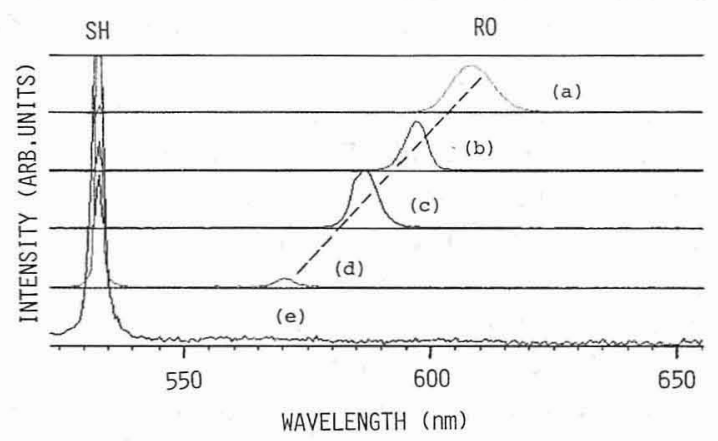

FIG. 2. Single-laser-shot RO spectra [(a)-(d)] from single RH-6G-doped sphere $\left(\sim 10^{-3} \mathrm{M}\right.$ concentration, $d \approx 210 \mu \mathrm{m}$ diam), by Nd:YAG SH $(532$ $\mathrm{nm}$ ) pumping, under nearly constant pumping intensities; (a) without PMMA seeding, (b), (c), and (d) ( 1/20-PMMA volume of the initial droplet volume, 1/10, and 1/5) with gradual increasing PMMA seeding in the droplets, and (e) with more PMMA seeding ( 1/2-PMMA volume of the initial droplet volume) than (d), which corresponds to fluorescence.

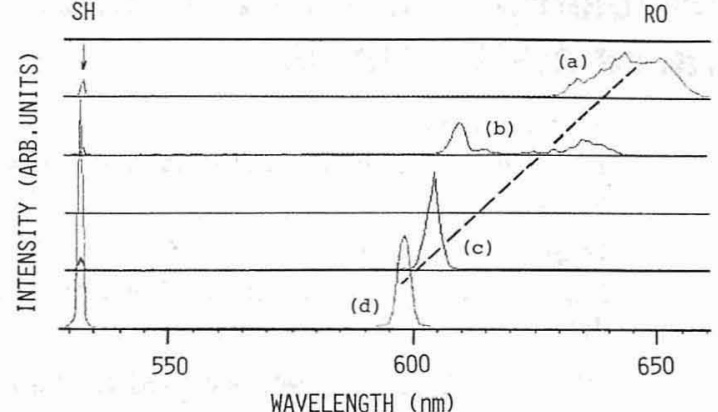

FIG. 3. Single-laser-shot RO spectra [(a)-(d)] from single RH-101-doped sphere $\left(\sim 10^{-3} \mathrm{M}, d \approx 150 \mu \mathrm{m}\right)$, by Nd:YAG SH (532 nm) pumping, under nearly constant pumping intensities; (a) without PMMA seeding, (b), (c), and (d) $(\sim 1 / 20,1 / 10$, and $1 / 5)$ with gradual increasing PMMA seeding in the droplets.

ing PMMA seeding in the droplets in Figs. 2(a), 2(b), and 2(c), respectively, and Fig. 2(e) ( 1/2-PMMA volume of the initial droplet volume) is the fluorescence spectrum [vertical gain is expanded compared with those of Figs. 2(a)-2(d)] with more PMMA seeding.

Figure 3 exhibits the similar characteristics to Fig. 2. Figure 3(a) comes from the RH-101-doped droplet $\left(\sim 10^{-3}\right.$ $\mathrm{M}, d \approx 150 \mu \mathrm{m})$ alone, Figs. 3(b), 3(c), and 3(d) $(\sim 1 / 20$, $1 / 10$, and $1 / 5$ ) correspond to the lasing spectrum with gradual increasing PMMA seeding in the droplets in Fig. 3(a), Fig. 3(b), and Fig. 3(c), respectively. In Figs. 2 and 3, there was no laser-induced breakdown, ${ }^{15}$ because the pumping intensities were lowered by the use of neutral-density filters.

In Figs. 2 (RH-6G) and 3 (RH-101), it is found that the lasing spectra in the unseeded droplets are situated in nearly the same spectral region as those (RH- $6 \mathrm{G}^{5}$ and $\left.\mathrm{RH}-101^{6}\right)$ in dye-doped solid spheres. The shorter wavelength limit of lasing in Figs. 2 and 3 seems to be restricted with absorption losses of the respective dyes; absorption loss in the respective dye-lasing region is situated in the shorter and longer wavelength regions in RH-6G and RH-101, respectively, resulting in the tuning range of the respective dyes.

We intend to give a simple physical meaning of the present experimental results (with the blueshifts in the dye lasing spectra resulting from the existence of scattering particles), by use of the gain-frequency (inverse-wavelength) characteristics calculated by Weber and Bass. ${ }^{16}$ The result is as follows (see Fig. 4): (1) The gain-frequency characteristics denote that the position of peak gain with increase of the excited-population shifts toward higher frequency (the shorter wavelength). (2) Laser emission arises at (angular) frequency $\omega$ when the laser gain $G(\omega)$ equals the cavity loss $L(\omega)$, which is determined by losses that result from reflection, diffraction, scattering, and absorption of the laser beam in the optical cavity. We deduce that low (or high) gain is necessary for lasing to satisfy the relation $G(\omega)=L(\omega)$ when the cavity has a high (or low) $Q$ value (HQ or LQ, respectively, in Fig. 4), where the corresponding frequency is low (or high). Consequently, dye lasing occurs at longer (or shorter) wavelengths when the cavity $Q$ value is high (or low). In addition, it is expected that the gradually increasing 


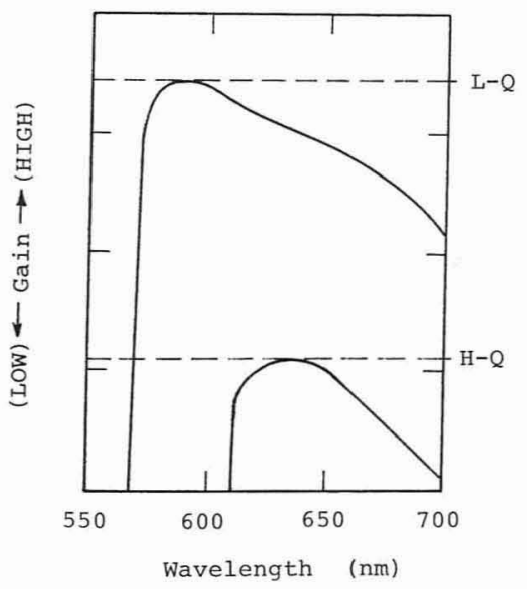

FIG. 4. Schematic diagram exhibiting a general relation of wavelength dependence on gain/loss curves in rhodamine $6 \mathrm{G}$ dye lasers, calculated by Weber and Bass, in which informations for other dyes are included (see Ref. 16).

PMMA seeding induces the gradually decreasing cavity $Q$ value as a result that the seeding particles obstruct propagation of a beam in the droplet, thereby reducing an effective optical pathlength. Because the cavity $Q$ value is proportional to mirror reflectivity and optical path length if the other parameters are kept constant, lasing characteristics at higher frequency (at shorter wavelength) with decrease of mirror reflectivity predicted and observed (see Fig. 8 in Ref. 16) is similar to those at shorter wavelengths with increase of the seeding particles in the present results. In the case of Fig. 2(e), the cavity $Q$ is completely spoiled. Thus we could give a simple explanation for the blueshifted lasing spectra as being due to the gradual decreases of the cavity $Q$ value (corresponding to the gradual increases of the scattering particles in the droplet cavity).

The spiky or complex band structure of the observed laser spectra [Figs. 3(a) and 3(b)] suggests that the droplets are exciting more than one MDR, accompanied by broadband emission that is due to the existence of some effective resonators with different $Q$ values. ${ }^{17}$ Even in the unseeded droplet [Fig. 3(a)], resonators with different $Q$ values might exist from the beginning, because this unseeded droplet has a spectrum that is different from the smooth spectrum of a different unseeded droplet [Fig. 2(a)].

It was confirmed, that the present blueshifted lasing spectra are not due to the decrease of the dye concentration as a result of dye degradation by successive laser pumping, from other experiments that seeding scattering PMMA particles into new (unseeded) dye-doped droplets also causes blueshifted lasing. Further, an analogous adsorption of RH-6G molecules- $\mathrm{RH}-6 \mathrm{G}$ precipitate onto the surface of the scattering particles ${ }^{18}$ and/or dissolution of the PMMA particles in the organic solvents may occur in the present case, thereby reducing the droplet dye concentration. The effect of these conjectured adsorption and dissolution of PMMA on the observed lasing spectra remains to be investigated; however, a method with $Q$-value variation that inserts artificial scatterers and then pulls back scatterers in turn into and then from the droplet may be a simple effective method of shift- ing the wavelength in spherical liquid dye lasers. The experimental result that the insertion and the pull back of a fine wire as a scatterer in the droplet cause the wavelength shifts toward shorter (by the insertion) and longer wavelengths (by the pull back; as it was in the unseeded droplet) supports the validity of this principle. Both conjectured effects, accompanied by reduced dye concentration as mentioned above, never cause the retracement toward foregoing lasing wavelengths. More improved future experiments for shifting wavelengths may give a clearer physical meaning to the mechanism of the scattering effects, ${ }^{18,19}$ as well as blueshifted lasing spectra with scatterers (in the present case and in Refs. 6, 10, and 18). In any case, wavelength shifts based on variations of cavity $Q$ values as a result of the insertion of artificial scattering particles seem to be one of the candidates for wavelength-tuning methods in spherical dye lasers.

In summary, we have reported the first experiments, to our knowledge, for wavelength tuning of droplet dye lasers. The experimental results of the wavelength tuning have been given, in principle, based on a cavity $Q$ value in terms of a general wavelength dependence of dye lasing determined by gain/loss characteristics, in the droplet dye lasers. We hope that the present technique may lead the basis for tunable microsphere dye lasers with high $Q$ values (low thresholds).

The authors gratefully acknowledge the continuous encouragements from Professor H. Inaba, Tohoku Institute of Technology.

${ }^{1}$ A. Biswas, H. Latifi, R. L. Armstrong, and R. G. Pinnick, Opt. Lett. 14, 214 (1989).

${ }^{2}$ H. Latifi, A. Biswas, R. L. Armstrong, and R. G. Pinnick, Appl. Opt. 29, 5387 (1990).

${ }^{3}$ A. J. Campillo, J. D. Eversole, and H.-B. Lin, Phys. Rev. Lett. 67, 437 (1991).

${ }^{4}$ M. K.-Gonokami, K. Takeda, H. Yasuda, and K. Ema, Jpn. J. Appl. Phys. 31, L99 (1992).

${ }^{5}$ H. Taniguchi, H. Yamada, T. Fujiwara, S. Tanosaki, H. Ito, H. Morozumi, and M. Baba, Jpn. J. Appl. Phys. 32, L58 (1993).

${ }^{6}$ H. Taniguchi, S. Tanosaki, H. Yamada, T. Fujiwara, and M. Baba, J. Appl. Phys. 73, 7957 (1993).

${ }^{7}$ R. G. Pinnick, A. Biswas, P. Chylek, R. L. Armstrong, H. Latifi, E. Creegan, V. Srivastava, M. Jarzembski, and G. Fernandez, Opt. Lett. 13, 494 (1988).

${ }^{8}$ W.-F. Hseih, J. B. Zheng, and R. K. Chang, Opt. Lett. 13, 496 (1988).

${ }^{9}$ A. Biswas, R. L. Armstrong, and R. G. Pinnick, Opt. Lett. 15, 1191 (1990).

${ }^{10} \mathrm{H}$. Taniguchi and S. Tanosaki, Jpn. J. Appl. Phys. 32, L1421 (1993).

${ }^{11} \mathrm{H}$. Taniguchi and H. Tomisawa, Rev. Sci. Instrum. 64, 3594 (1993).

${ }^{12}$ R. L. Armstrong, J.-G. Xie, T. E. Ruekgauer, and R. G. Pinnick, Opt. Lett. 17, 943 (1992).

${ }^{13}$ R. L. Armstrong, J.-G. Xie, T. E. Ruekgauer, J. Gu, and R. G. Pinnick, Opt. Lett. 18, 119 (1993).

${ }^{14}$ J.-G. Xie, T. E. Ruekgauer, R. L. Armstrong, and R. G. Pinnick, Opt. Lett. 18, 340 (1993).

${ }^{15}$ A. Biswas, R. G. Pinnick, J.-G. Xie, T. E. Ruekgauer, and R. L. Armstrong, Opt. Lett. 17, 1569 (1992).

${ }^{16}$ M. J. Weber and M. Bass, IEEE J. Quantum Electron. QE-5, 175 (1969).

${ }^{17}$ A. M. Korobov, V. N. Uranov, V. A. Shcheglov, and M. I. Dzyubenko, Opt. Commun. 9, 336 (1973).

${ }^{18}$ M. Essien, R. L. Armstrong, and J. B. Gillespie, Opt. Lett. 18, 762 (1993).

${ }^{19}$ H.-B. Lin, A. L. Huston, J. D. Eversole, A. J. Campillo, and P. Chylek, Opt. Lett. 17, 970 (1992). 